\title{
Los factores ambientales y su influencia en los patrones de actividad física en adolescentes. \\ The environmental factors and their influence in the patterns of physical activity in adolescents.
}

\author{
Zaragoza Casterad, J avier \\ Facultad de Ciencias Humanas y de la Educación. Universidad de Zaragoza \\ Serra Puyal, José Ramón \\ Facultad de Salud y Deporte. Universidad de Zaragoza \\ Ceballos Gurrola, Oswaldo \\ Facultad de Organización Deportiva. Universidad Autónoma de Nuevo León (Méjico) \\ Generelo Lanaspa, Eduardo \\ Facultad de Salud y Deporte. Universidad de Zaragoza \\ Serrano Ostariz, Enrique \\ Escuela Universitaria de Ciencias de la Salud. Universidad de Zaragoza \\ Julián Clemente, J osé Antonio \\ Facultad de Ciencias Humanas y de la Educación. Universidad de Zaragoza
}

\section{Resume n}

La mayoría de estudios demuestran; a) un descenso de la actividad física (AF) que coincide con la adolescencia, sobre todo en población femenina, b) durante la primavera y la jornada escolar se realiza mayor AF que durante el invierno o el fin de semana, siendo esta actividad fundamentalmente moderada. El objetivo de este estudio es comparar los niveles de actividad física de los adolescentes de dos poblaciones aragonesas (Zaragoza y Sabiñánigo). Para ello hemos utilizado el "Four by one-day Physical Activity Questionnaire". Los resultados de nuestro estudio señalan que: el gasto energético total derivado de la práctica de actividad física, es mayor en la zona rural estudiada; mientras que el gasto energético derivado de la jornada escolar tanto de invierno como de primavera es mayor en la población urbana estudiada, el gasto energético derivado del fin de semana tanto de invierno como de primavera es mayor en la población rural; es mayor la actividad física realizada por los hombres que por las mujeres solo en el caso de la población urbana estudiada; predominan las actividades ligeras o muy ligeras en ambos sexos y poblaciones; encontramos una relación significativa entre las horas que ven televisión y la práctica de AF.

Palabras clave: Adolescencia, Niveles de actividad física, Gasto energético, promoción de la actividad física Key Words: Teenagers, Levels of physical activity, energy expenditure, physical activity promotion.

Correspondencia: Dr. Zaragoza Casterad Javier.

Facultad de Ciencias Humanas y de la Educación. Universidad de Zaragoza.

E-mail: zaragoza@unizar.es 


\title{
The environmental factors and their influence in the patterns of physical activity in adolescents.
}

\section{Los factores ambientales y su influencia en los patrones de actividad física en adolescentes.}

\author{
Zaragoza Casterad, J avier \\ Facultad de Ciencias Humanas y de la Educación. Universidad de Zaragoza \\ Serra Puyal, José Ramón \\ Facultad de Salud y Deporte. Universidad de Zaragoza \\ Ceballos Gurrola, Oswaldo \\ Facultad de Organización Deportiva. Universidad Autónoma de Nuevo León (Méjico) \\ Generelo Lanaspa, Eduardo \\ Facultad de Salud y Deporte. Universidad de Zaragoza \\ Serrano Ostariz, Enrique \\ Escuela Universitaria de Ciencias de la Salud. Universidad de Zaragoza \\ Julián Clemente, J osé Antonio \\ Facultad de Ciencias Humanas y de la Educación. Universidad de Zaragoza
}

\begin{abstract}
Most of studies they demonstrate; a) a reduction of the physical activity (AF) that agrees with the adolescence, mainly in feminine population, b) during the spring and the scholastic day is made greater high frequency than during the winter or the weekend, being this fundamentally moderate activity. The objective of this study is to compare the levels of physical activity of the adolescents of two Aragonese populations (Zaragoza and Sabiñánigo). For it we have used the "Four by one-day Physical Activity Questionnaire". The results of our study indicate that: the energy expenditure total ( kcal/kg/day) derivative of the

practice of physical activity, is greater in the studied countryside; whereas the energy expenditure derived from the scholastic day as much of winter as of spring is greater in the studied urban population, the energy expenditure derived from the weekend as much of winter as of spring he is greater in the rural population; the physical activity made by the men is greater who by the women single in the case of the studied urban population; the slight or very slight activities in both sexes and populations predominate; we found a significant relation between the hours that watch and the high frequency practice.
\end{abstract}

Palabras clave: Adolescencia, Niveles de actividad física, Gasto energético, promoción de la actividad física Key Words: Teenagers, Levels of physical activity, energy expenditure, physical activity promotion.

Correspondence: Dr. Zaragoza Casterad Javier.

Facultad de Ciencias Humanas y de la Educación. Universidad de Zaragoza.

E-mail: zaragoza@unizar.es 


\section{Introducción}

Los estilos de vida saludables (EVS) son patrones de conducta que resultan beneficiosos para la salud. Estos patrones son aprendidos gracias a la influencia de los padres, compañeros, amigos, o por la influencia de la escuela, los medios de comunicación Los factores ambientales como la disponibilidad de infraestructuras, la oferta de programas, la accesibilidad a entornos naturales..., pueden tener influencia en la conducta activa del adolescente. Parece que hay unanimidad en la literatura científica en que las características geográficas, el clima, las costumbres..., pueden facilitar o dificultar la práctica de AF. La influencia de los otros significativos en interacción con el medio físico y social, representa un factor determinante del estilo de vida adolescente. Además, las conductas que conforman los EVS, están relacionados entre sí (Elliot, 1993; Pate et al., 1996; Pastor et al., 1998), sobre todo en edad joven.

La adolescencia es una etapa crítica en el aprendizaje de conductas saludables, entre las que se encuentra la práctica de AF. Es evidente, por otro lado, que estamos ante un momento crítico en tanto que los hábitos desarrollados durante esta etapa, pueden continuar durante la edad adulta (Livingstone, 1994; Meredith et al., 1991; Sallis et al., 1991; Taylor et al., 1999).

Cada vez conocemos con mayor evidencia que el sedentarismo no solo afecta a la población adulta, sino también a la infancia y a la adolescencia. Diferentes estudios, (Wold, 1989; Sánchez, et al., 1992; Sallis, 1993; Mendoza et al., 1994; Sánchez-Barrera et al., 1995; Cantón y Sánchez 1997; Castillo y Balaguer 1998; Ponseti et al., 1998; Casimiro, 1999; Rodríguez, 2000; Pérez y Delgado 2002; Ministerio de Sanidad y Consumo, 2003), demuestran el descenso de práctica de AF en la población infantil y juvenil, siendo este descenso más acusado en población femenina.

Los cambios socioculturales acaecidos en las comunidades urbanas postmodernas, generan nuevas formas de vida que afectan a la consideración que se tiene de diferentes conductas, entre las que se encuentra la práctica de AF, (Tercedor et al., 2003). En palabras de Olivera $(2005,3)$, hemos pasado "del hombre nómada, hambriento, creyente y cazador, al hombre sedentario, sobrealimentado, descreído y consumista”.

Una de las graves consecuencias de este sedentarismo, es el aumento de la obesidad infantil. La AF puede contribuir a prevenirla, sobre todo teniendo en cuenta que la restricción calórica en estas edades, puede afectar al crecimiento y a la maduración (Williams, 1986). 
Este trabajo y siguiendo las fases del estudio de la AF que propone Sallis (1995), se sitúa en la segunda fase , y pretende conocer a) cuales son los niveles de actividad física habitual de una muestra de sujetos adolescentes que cursan Educación Secundaria Obligatoria (E.S.O.), de dos zonas diferenciadas de la Comunidad Autónoma de Aragón. Concretamente una zona rural de la provincia de Huesca (Sabiñánigo), ciudad cercana a los 10.000 habitantes, enclavada en el Pirineo Aragonés que ha sido tradicionalmente industrial, pero que en la actualidad está siendo objeto de una reconversión hacia el sector servicios, y otra zona urbana (ciudad de Zaragoza), con una población cercana a los 650.000 habitantes, y b) analizar los factores que influencian esta práctica, al objeto de establecer intervenciones intersectoriales, que faciliten la instauración de hábitos saludables en el estilo de vida presente y futuro de los adolescentes de referencia.

\section{Material y métodos}

\section{Sujetos}

Del total de los adolescentes escolarizados en Educación Secundaria Obligatoria de ambas poblaciones, se toma una muestra estratificada, con fijación proporcional a las variables de interés. La muestra total está constituida por 470 adolescentes, de los cuales 394 pertenecen a la ciudad de Zaragoza (195 mujeres y 199 hombres) y 76 alumnos de Sabiñánigo (43 mujeres y 33 hombres), de una edad media de 13,2 \pm 2,4 años.

\section{Instrumentos}

Utilizamos el cuestionario denominado "Four by one-day physical activity questionnaire" (Cale, 1994). Es un cuestionario de recuerdo que permite estimar la actividad física total, o gasto energético (GE) dentro y fuera del ámbito escolar. El instrumento recoge la actividad del día anterior y lo hace en cuatro ocasiones; dos en temporada de invierno y dos en primavera. Dos registros se realizarán en días escolares y otros dos en fin de semana. Contiene una lista de actividades, divididas en periodos cronológicos para favorecer el recuerdo.

La recogida de datos se hace de forma autoadministrada en presencia de un investigador aprovechando un momento de clase, siempre en presencia del tutor del grupo o del profesor de Educación Física. Cada actividad se asocia a un gasto energético (Ainsworth et al. 1993). Los datos se expresarán como GE en kcal/kg/día. 
Zaragoza, J., Serra, J.R., Ceballos, O., Generelo, E., Serrano, E., Julian, J.A. (2006). Los Factores ambientales y su influencia en los patrones de actividad física en adolescentes. Revista Internacional de Ciencias del Deporte. 4 (2), 1-14. http://www.cafyd.com/REVISTA/art1n4a06.pdf

Las actividades están divididas, siguiendo a Blair et al., (1994) en: dormir (1 kcal/kg/día); actividades muy ligeras (entre 1,5-4 kcal/kg/día); actividades moderadas (entre 4-6 $\mathrm{kcal} / \mathrm{kg} / \mathrm{día}$ ); actividades vigorosas (más de $6 \mathrm{kcal} / \mathrm{kg} /$ día). Clasificamos a los sujetos en cuatro categorías o niveles de AF (Sallis, 1993): activo (40 o más kcal/kg/día); moderadamente activo (entre 36,9 y 39,99 kcal/kg/día); inactivo (entre 33 y 36,99 $\mathrm{kcal} / \mathrm{kg} / \mathrm{día}$ ); muy inactivo (menos de $33 \mathrm{kcal} / \mathrm{kg} / \mathrm{día}$ ).

Para obtener resultados se utilizará una estadística descriptiva, utilizando el programa estadístico SPSS 13.0. Hemos realizado por un lado, estadísticos descriptivos de todas las variables estudiadas. En las variables cuantitativas, la media y desviación estándar. En las variables cualitativas, frecuencias absolutas y porcentajes. Por otro lado, hemos efectuado el contraste de hipótesis para medias para comprobar si existen diferencias significativas entre grupos. Hemos aplicado el test de la t de Student y se ha verificado la hipótesis de igualdad de varianzas con el test de Levene. Para comprobar si existe asociación entre el tiempo viendo televisión y el nivel de AF, utilizamos el coeficiente de correlación de Pearson.

\section{Resultados}

Encontramos diferencias significativas $(\mathrm{p}<0,05)$ en las poblaciones estudiadas en cuanto al, GE medio (GEM), GE durante el fin de semana invierno (GEFI), GE de la jornada escolar de invierno (GEJEI), GE de la jornada escolar de primavera (GEJEP), y del número de horas viendo la televisión. Los adolescentes de la ciudad de Zaragoza presentan mayor GEJEI (38,27 kcal/kg/día) y mayor GEJEP (37,04 kcal/kg/día), mientras que la población de Sabiñánigo presenta mayor GEM (39,17 kcal/kg/día), mayor gasto energético durante el fin de semana de invierno (GEFI) (38,87 kcal/kg/día) y de primavera (GEFP) (41,74 $\mathrm{kcal} / \mathrm{kg} / \mathrm{día})$. 
Zaragoza, J., Serra, J.R., Ceballos, O., Generelo, E., Serrano, E., Julian, J.A. (2006). Los Factores ambientales y su influencia en los patrones de actividad física en adolescentes. Revista Internacional de Ciencias del Deporte. 4 (2), 1-14. http://www.cafyd.com/REVISTA/art1n4a06.pdf

Tabla 1. Niveles de AF (kcal/kg/día)

\begin{tabular}{|c|c|c|c|}
\hline \multirow{2}{*}{ VARIABLES } & ZARAGOZA & SABIÑÁNIGO & \multirow{2}{*}{ P } \\
\cline { 2 - 4 } & Media (DE) & $39,17(5,10)$ & $<0,05$ \\
\hline \multirow{2}{*}{ GEM } & $37,66(2,95)$ & $34,39(5,10)$ & $<0,05$ \\
\hline GEJEI & $38,27(4,81)$ & $30,86(6,01)$ & $<0,05$ \\
\hline GEJEP & $37,04(3,64)$ & $38,87(9,54)$ & $<0,05$ \\
\hline GEFI & $35,95(5,09)$ & $41,74(7,67)$ & $<0,05$ \\
\hline GEFP & $39,44(5,36)$ & $35,63(5,60)$ & $<0,05$ \\
\hline GEI & $37,11(3,82)$ & $35,30(3,76)$ & $<0,05$ \\
\hline HORAS DIARIAS TV & $2,50(1,01)$ & $1,40(0,58)$ & $<0,05$ \\
\hline
\end{tabular}

GEM: gasto energético medio; GEJ El: gasto energético jornada escolar invierno; GEJ EP: gasto energético jornada escolar primavera; GEFI: gasto energético fin de semana invierno; GEFP: gasto energético fin de semana primavera; GEI: gasto energético invierno; GEP: gasto energético primavera.

Solo encontramos diferencias significativas en cuanto a las categorías de intensidad de la $\mathrm{AF}$, en el género masculino de ambas poblaciones. Concretamente los sujetos de género masculino de Sabiñánigo duermen más tiempo, mientras que los sujetos de género masculino de la ciudad de Zaragoza, realizan más AF de carácter muy ligero y vigoroso que los escolares de la ciudad de Sabiñánigo $(\mathrm{p}<0,05)$.

Tabla 2. Niveles de AF (kcal/kg/día). según categorías de intensidad y género

\begin{tabular}{|c|c|c|c|c|c|c|}
\hline & \multicolumn{2}{|c|}{ HOMBRES } & \multirow[b]{2}{*}{$\mathbf{P}$} & \multicolumn{2}{|c|}{ MUJERES } & \multirow[b]{2}{*}{$\mathbf{P}$} \\
\hline & $\begin{array}{c}\mathrm{Z} \\
\text { Media } \\
\text { (DE) }\end{array}$ & $\begin{array}{c}\text { S } \\
\text { Media } \\
\text { (DE) }\end{array}$ & & $\begin{array}{c}\mathrm{Z} \\
\text { Media (DE) }\end{array}$ & $\begin{array}{c}\text { S } \\
\text { Media (DE) }\end{array}$ & \\
\hline Dormir & $\begin{array}{c}8,89 \\
(0,75)\end{array}$ & $\begin{array}{l}9,80 \\
(3,5\end{array}$ & $<0,05$ & $\begin{array}{c}9,03 \\
(0,66)\end{array}$ & $\begin{array}{l}9,44 \\
(3,0)\end{array}$ & $>0,05$ \\
\hline Muy ligeras & $\begin{array}{l}17,67 \\
(1,86)\end{array}$ & $\begin{array}{c}15,91 \\
(4,1)\end{array}$ & $<0,05$ & $\begin{array}{l}17,21 \\
(1,89)\end{array}$ & $\begin{array}{c}16,84 \\
(1,9)\end{array}$ & $>0,05$ \\
\hline Ligeras & $\begin{array}{c}5,44 \\
(2,36)\end{array}$ & $\begin{array}{l}5,74 \\
(2,8)\end{array}$ & $>0,05$ & $\begin{array}{c}6,49 \\
(2,30=\end{array}$ & $\begin{array}{l}6,23 \\
(2,7)\end{array}$ & $>0,05$ \\
\hline Moderadas & $\begin{array}{c}2,41 \\
(1,63)\end{array}$ & $\begin{array}{l}2,48 \\
(3,9)\end{array}$ & $>0,05$ & $\begin{array}{c}2,78 \\
(1,87)\end{array}$ & $\begin{array}{l}1,92 \\
(2,6)\end{array}$ & $>0,05$ \\
\hline Vigorosas & $\begin{array}{c}7,82 \\
(2,99)\end{array}$ & $\begin{array}{l}6,46 \\
(2,5)\end{array}$ & $<0,05$ & $\begin{array}{c}3,96 \\
(2,90)\end{array}$ & $\begin{array}{l}3,89 \\
(4,3)\end{array}$ & $>0,05$ \\
\hline
\end{tabular}

Z: Zaragoza; S: Sabiñánigo; 
Zaragoza, J., Serra, J.R., Ceballos, O., Generelo, E., Serrano, E., Julian, J.A. (2006). Los Factores ambientales y su influencia en los patrones de actividad física en adolescentes. Revista Internacional de Ciencias del Deporte. 4 (2), 1-14. http://www.cafyd.com/REVISTA/art1n4a06.pdf

El porcentaje de sujetos catalogados como inactivos o muy inactivos, como se puede apreciar en la tabla 3 , es mayor en la población de Zaragoza $(47,2 \%)$ respecto a la población de Sabiñánigo (39\%).

Tabla 3. Porcentajes de la población según su nivel de actividad física.

\begin{tabular}{|c|c|c|c|c|}
\hline \multirow{2}{*}{ CATEGORÍAS } & \multicolumn{2}{|c|}{ Zaragoza } & \multicolumn{2}{c|}{ Sabiñánigo } \\
\cline { 2 - 5 } & $\mathbf{N}$ & $\mathbf{\%}$ & $\mathbf{N}$ & $\mathbf{\%}$ \\
\hline ACTIVO & 76 & 19,3 & 21 & 28 \\
\cline { 2 - 5 } MODERADAMENTE ACTIVO & 132 & 33,5 & 25 & 33 \\
\cline { 2 - 5 } INACTIVO & 184 & 46,7 & 30 & 39 \\
\cline { 2 - 5 } MUY INACTIVO & 2 & 0,5 & 0 & 0 \\
\hline
\end{tabular}

Considerando ambos géneros y poblaciones, encontramos que en la población de Sabiñánigo, el porcentaje de mujeres activas (42\%) es mayor que el porcentaje de hombres (18\%), mientras que en la población de Zaragoza, el porcentaje de hombres activos (29,1\%) es superior al porcentaje de mujeres activas (5,6\%), (tabla 4).

Tabla 4. Porcentaje de la población según niveles de actividad física y género.

\begin{tabular}{|c|c|c|c|c|c|c|c|c|}
\hline & \multicolumn{4}{|c|}{ HOMBRES } & \multicolumn{4}{|c|}{ MUJERES } \\
\hline & \multicolumn{2}{|c|}{$\mathbf{Z}$} & \multicolumn{2}{|c|}{$\mathbf{S}$} & \multicolumn{2}{|c|}{$\mathbf{Z}$} & \multicolumn{2}{|c|}{$\mathbf{S}$} \\
\hline & $\mathbf{N}$ & $\%$ & $\mathbf{N}$ & $\%$ & $\mathbf{N}$ & $\%$ & $\mathbf{N}$ & $\%$ \\
\hline ACTIVO & 58 & 29,1 & 8 & 18 & 11 & 5,6 & 13 & 42 \\
\hline $\begin{array}{c}\text { MODERADAMENTE } \\
\text { ACTIVO }\end{array}$ & 63 & 31,6 & 12 & 27 & 72 & 36,9 & 13 & 42 \\
\hline INACTIVO & 77 & 38,8 & 25 & 55 & 112 & 57,5 & 5 & 16 \\
\hline MUY INACTIVO & 1 & 0,5 & 0 & 0 & 0 & 0 & 0 & 0 \\
\hline
\end{tabular}

Z: Zaragoza; S: Sabiñánigo.

Los adolescentes de la ciudad de Zaragoza invierten más tiempo en ver la televisión (2,50 $\pm 1,01$ horas) que los adolescentes de Sabiñánigo (1,40 $\pm 0,58$ horas), siendo estas diferencias estadísticamente significativas. En ambas poblaciones encontramos relación, negativa, débil pero significativa $(\mathrm{p}<0,05)$, entre el tiempo dedicado a ver la televisión $\mathrm{y}$ los niveles de AF (en la población de Zaragoza la correlación fue de $\mathrm{r}=-, 14$, y en la población de Sabiñánigo fue de $r=-, 21$ ). 


\section{Discusión}

Existe una enorme preocupación por el nivel de sedentarismo de la población adolescente. Diferentes estudios señalan el importante descenso de la práctica de AF en este subgrupo, con relación a etapas anteriores (Sánchez 1990; Thirlaway et al, 1993; Savage et al., 1998; Ministerio de Sanidad y Consumo, 2003; Miquel et al., 2004). Existen según Sallis (1995), diferentes variables que influencian esta práctica: variables personales (biológicas y psicológicas) y ambientales (sociales y físicas). Las variables ambientales entre las que se encuentra el entorno físico son una de las variables susceptibles de ser modificadas que pueden influenciar la realización de AF.

El GEM de las poblaciones estudiadas, es similar al encontrado en otros trabajos que han utilizado esta misma metodología, (Cantera, 1997; Cale 1994; Miquel et al., 2004). El elevado nivel de sedentarismo (en nuestro caso por encima del 30\% en ambas poblaciones), es mayor en la población urbana de Zaragoza. Los resultados de estudios realizados sobre la influencia del entorno, en la realización de AF, son contradictorios. Por un lado, encontramos trabajos que no encuentran diferencias significativas en los adolescentes de entornos rurales y urbanos (Shepard et al., 1980). Otros trabajos certifican que los jóvenes de medio urbano, realizan mayor AF que los de medio rural (Cantera, 1997). Nuestros resultados en cambio, señalan mayores niveles de AF en la población rural estudiada.

A nivel general se acepta que los adolescentes de género masculino realizan mayor práctica de AF que los de género femenino (Verschuur et al., 1985; Sallis et al., 2000; Gavarry et al 2003; Riddoch et al., 2004; Montil, 2004). Así se pone de manifiesto en nuestra investigación, pero solo en el caso de la población zaragozana, ya que los adolescentes de género masculino de la población de Sabiñánigo, son más inactivos que los de género femenino. Las diferencias encontradas en la conducta de hacer AF relativas al género, pueden depender de la medida utilizada (Sallis et al, 1998) y de los criterios utilizados para diferenciar entre actividad y sedentarismo (Riddoch et al., 1995).

Algunas investigaciones (Cantera, 1997; Pate et al., 1994; Herva et al., 1994), señalan que en época invernal es menor la práctica de AF que durante la primavera, datos que solo corroboramos en nuestra investigación, en el caso de los adolescentes de la ciudad de Zaragoza.

No existe unanimidad a la hora de definir si se hace más práctica de AF durante el fin de semana o durante la jornada escolar. Autores como Gavarry et al., (2003), Gilvey et al., (1995), Sallo et al., (1997), no encuentran diferencias entre ambos períodos, mientras que otros estudios (Trost et al., 2000; Falgairette, 1996), señalan que los adolescentes realizan mayor AF durante la jornada escolar. Nuestro estudio demuestra que la mayor AF se realiza durante el fin de semana, solo en la población rural de Sabiñánigo. 
Zaragoza, J., Serra, J.R., Ceballos, O., Generelo, E., Serrano, E., Julian, J.A. (2006). Los Factores ambientales y su influencia en los patrones de actividad física en adolescentes. Revista Internacional de Ciencias del Deporte. 4 (2), 1-14. http://www.cafyd.com/REVISTA/art1n4a06.pdf

El mayor GEFI en la población rural estudiada, podría estar influenciado, entre otros factores, por las diferentes características del entorno físico donde se sitúan estas poblaciones (posibilidad que ofrece el entorno de las estaciones de ski, muy cercanas a la población de Sabiñánigo), corroborando conclusiones de investigaciones (Klesges et al., 1990; Ross et al., 1985), sobre la práctica de AF en adolescentes, y que concluyen señalando que la accesibilidad a las instalaciones y programas de actividades, el tiempo que pueden estar en la calle..., pueden estar relacionados con la conducta de hacer AF, incluso con su frecuencia e intensidad (Sallis et al., 1997).

El GE de la población estudiada se deriva principalmente de las actividades muy ligeras y ligeras, a diferencia de otros estudios que encuentran que en la intensidad de la práctica adolescente, existe un predominio de la AF moderada (Montil 2004). Los sujetos estudiados de ambas poblaciones, presentan un GE derivado de las actividades vigorosas que está por debajo de los resultados obtenidos en otros trabajos (Cantera, 1997; Shepard, 1980), y en sintonía con otras investigaciones, donde se señala que un gran porcentaje de la población joven, no realiza actividad física vigorosa (Mendoza, 1994). Al igual que en otros trabajos (Stephens, 1985), los sujetos de género masculino de nuestro estudio, realizan más AF de tipo vigoroso que los sujetos de género femenino.

El tiempo dedicado a ver la televisión en nuestro estudio, está en sintonía con los resultados de otros trabajos que señalan que los niños y jóvenes dedican una media de 2-3h diarias (Hernández et al., 1999; Lindquist et al., 1999). Estos datos pueden entenderse en tanto en cuanto, en el caso de nuestro país, una de las actividades de tiempo libre realizadas con mayor frecuencia por la población española mayor de 15 años, es ver la televisión, que es realizada prácticamente todos los días, (Ministerio de Educación, Cultura y Deporte, 2001).

Encontramos resultados contradictorios respecto a la influencia o relación de las horas dedicadas a ver televisión y los niveles de AF. Por un lado, encontramos trabajos que afirman que el ocio tecnológico es uno de los factores que pueden influenciar la dedicación a la práctica de AF (Kimm et al., 1996; Shannon et al., 1991; Bernard et al., 1995;). Por otro lado, existen estudios que no encuentran ninguna relación (Tucker, 1990; Cantera, 1997; Biddle, 2003). Estas discrepancias pudieran ser debidas, al igual que hemos manifestado anteriormente, a los diferentes criterios utilizados a la hora de clasificar el grado de sedentarismo de la población adolescente. 


\section{Conclusiones}

Como conclusiones principales, podemos señalar que:

- Mayor nivel de sedentarismo en la población urbana de Zaragoza.

- La inactividad es más acusada en la población rural, en el caso de los adolescentes de género masculino.

- Mientras que la población adolescente de Zaragoza realiza mayor AF durante la jornada escolar, la población de Sabiñánigo realiza mayor AF durante el fin de semana de invierno.

- Encontramos relación débil y negativa entre las horas que los adolescentes se implican en ver televisión y la realización de AF.

Al margen de las conclusiones prioritarias de este estudio y como valoración final, nos atrevemos a sugerir que muchos de los conocimientos derivados en este tipo de estudios, parecen insuficientes a la hora de que su aplicación genere pautas de intervención desde políticas institucionales. Parece conveniente recomendar que estos estudios se combinen con análisis de las influencias que determinan la práctica de actividad física en las poblaciones de referencia.

\section{Referencias bibliografícas}

Ainsworth, B.A., Haskell, WL., Leon, A.S. Jacobs, D.R. Montoye, H.J. Sallis, J.F. et al. (1993). Compendiun of physical activities: classification of energy costs of human physical activities. Medicine and Science in Sports and Exercise, 25 (1), 71-80.

Bernard, L., Lavalle, C. (1995). Overweight in Cree schoolchildren and adolescents associated with diet, low phyical activity, and high televisión Beijing. Journal of the American Dietetic Association, 95, (7), 800.

Blair, S.N., Meredith, M.D. (1994). The exercise health relationship. Does it apply to children and in youth?. En R.R. Pate., R.C. Hohm (Eds). Health and Fitness through physical education. Human Kinetics. Champaign, IL, 11-19.

Biddle, S. (2003). Social Psychology of physical activity and sedentary behaviour in young people. Revista Portuguesa de Ciências do Desporto, 3 (2), S15-S16.

Cale, L. (1994). Self-report measures of children's physical activity: recommendations for future development and a new alternative measure. Health Education Journal, $53,439-53$.

Cantera, M. A. (1997). Niveles de actividad física en la adolescencia. Estudio realizado en la población escolar de la provincia de Teruel. Tesis doctoral. Zaragoza: Universidad de Zaragoza. 
Cantón, E. y Sánchez, M. C. (1997). Deporte y calidad de vida: motivos y actitudes en una muestra de jóvenes valencianos. Revista de Psicología del Deporte, 12, 119135.

Casimiro, A. J. (1999). Comparación, evolución y relación de hábitos saludables y nivel de condición física-salud en escolares, al finalizar los estudio de Educación Primaria (12 años) y de Educación Secundaria Obligatoria (16 años). Tesis Doctoral.

Castillo, I. y Balaguer, I. (1998). Patrones de actividades físicas en niños y adolescentes. Apunts: Educación Física y Deportes, 54, 22-29.

Elliot, E.S. (1993). Health-enhancing and health-compromising lifestyles. En S.G. Millstein, A.C.Petersen y E.O. Nightingale (Eds). Promoting the health of adolescents. New directions for the twenty-first century. Oxford: Oxford University Press.

Falgairette, G. (1996). Evaluation of habitual physical activity from a week's heart rate monitoring in French school children. Europe Journal Applied Physiology, 74 (1-2), 153-161.

Gavarry, O., Giacomoni, M., Bernard, T., Seymat, M., y Falgairette, G. (2003). Habitual physical activity in children and adolescents during school and free days. Medicine and Science and in Sports and Exercise, 35 (3), 525-531

Gilbey, H., y Gilbey, M. (1995). The physical activity of Singapore primary children as estimated by heart rate monitoring. Pediatric Exercise Science, 7, 26-35.

Hernández, B., Gortmaker, S. L., Colditz, G. A., Peterson, K. E., Laird, N. M., y ParraCabrera, S. (1999). Association of obesity with physical activity, television programs and other forms of video viewing among children in Mexico City. International J ournal of Obesity and Related Metabolic Disorders, 23, 845-854.

Herva, H., Vuolle, P. (1994). Trenes in the use of time for physical activity in Finland and other countries. British Journal of Sport Medicine, 30, 84-89.

Huang, Y. C., y Malina, R. (1996). Physical activity and correlates of estimated energy expenditure in Taiwanese adolescents 12-14 years of age. American Journal of Human Biology, 8, 225-236.

Kimm, S. Y. S., y Kwiterovich, P. O. (1995). Childhood prevention of adult chronic diseases: rationale and strategies. En L. N. Y. Cheung y J. B. Richmond (Eds.), Child health, nutrition, and physical activity. Champaign, II: Human Kinetics.

Linquist, C. H., Reynolds, K. D., y Goran, M. I. (1999). Sociocultural determinants of physical activity among children. Preventive Medicine, 29, 305-312.

Livingstone, M. B. (1994). EE and physical activity in relation to fitness in children. Proceedings of the Nutrition Society, 53, 207-221. 
Mendoza, R., Sagrera, M. R., y Batista, J. M. (1994). Conductas de los escolares españoles relacionadas con la salud, 1986-1990. Madrid: Consejo Superior de Investigaciones Científicas.

Meredith, C. M., y Dwyer, J. T. (1991). Nutrition and exercise: effects on adolescent health. Annual Review Public Health, 12, 309-333.

Ministerio de Educación, Cultura y Deporte. (2001). Los españoles y el deporte: prácticas y comportamientos en la última década del siglo XX. Encuesta sobre los hábitos deportivos de los españoles, 2000. Madrid. Ministerio de Educación, Cultura y Deporte.

Ministerio de Sanidad y Consumo (2003). Encuesta Nacional de Salud. (2001). Madrid: Ministerio de Sanidad y Consumo.

Montill, M. (2004). Determinantes de la conducta de actividad física en población infantil. Tesis Doctoral. Universidad Politécnica de Madrid.

Olivera, J. (2005). Contra natura: el hombre contra el hombre. Apunts de Educación Física y Deportes 80, 1-4.

Pastor, Y., Balaguer I, García Merita, M.L: (1998). Dimensiones del estilo de vida relacionado con la salud en la adolescencia. Revista de Psicología General y Aplicada, 51 (3-4), 469-483.

Pate, R.R. Heath, G.W., Dowda, M., Trost, S.G. (1996). Associations between physical activity and other health behaviours in a representative sample of US adolescents. American Journal of Public Health, 86 (11), 1577-1581.

Pate, R.R., Long, B.J., Heath, G. (1994). Descriptive Epidemiology of physical activity in adolescents. Pediatric Exercise Science, 6, 434-447.

Pérez, I. J. y Delgado, M. (2002, Marzo). Modificaciones del interés, motivaciones y comportamientos de hábitos de tiempo libre y físico-deportivos tras un programa de intervención en Secundaria desde la E. F. orientada a la salud. Actas del II Congreso de Ciencias del Deporte. INEF, Madrid.

Ponseti, F. X., Gili, M., Palou, P., y Borrás, P. A. (1998). Intereses, motivos y actitudes hacia el deporte en adolescentes: Diferencias en función del nivel de práctica. Revista de Psicología del Deporte, 7 (2), 259-274.

Riddoch, C. J., Andersen, L., Weddeerkopp, N., Harro, M., Klasson-Heggebo, L., Sardinha, L. B., Cooper, A. R., y Ekelund, U. (2004). Physical activity levels and patterns of 9-and 15-yr- old European children. Medicine and Science and in Sports and Exercise, 36 (1), 86-92.

Rodríguez, A. (2000). Adolescencia y Deporte. Oviedo: Nobel. 
Ross, J.G., Dotson, C.O., Gilbert, G.G., Katz, S.J. (1985). After physical education... Physical outside of school education programs. Journal of Physical Education and Dance, 56 (1), 77-81.

Miquel Salgado-Araujo, J.L., Devís, J. (2003). La actividad física de los escolares (1216 años) de la Comunidad Valenciana: gasto energético y niveles de actividad. En III Congreso de la Asociación Española de Ciencias del Deporte. Valencia, 2004.

Sallis, J.F.(1993). Epidemiology of physical activity and fitness in children and adolescents. Critical Reviews in Food Science and Nutrition, 33, 403-408.

Sallis, J. F. (1995). A behavioural perspective on children's physical activity. En L. W. Y. Cheung, y J. B. Richmond (Eds.), Child health, nutrition, and physical activity. Champaign, Il: Human Kinetics.

Sallis, J. F. y Owen, N. (1997). Ecologic models. En K. Glanz, F. M. Lewis y B. K. Rimer (Eds.), Health behaviour and health education: theory, research, and practice ( $p p$ 403-424). San Francisco, CA, Jossy-Bass.

Sallis, J.F., Mckneize, T.LO: Elder, J.P., Hoy, P.L:, Galati, T., Berry, C., et al. (1998). Sex and athnic differences in children's physical activity: discrepancies between self-report and objective measures. Pediatric Exercise Science, 10, 277-284.

Sallis, J. F., Prochaska, J. J., y Taylor, W. C. (2000). A review of correlates of physical activity of children and adolescents. Medicine and Science and in Sports and Exercise, 32, 963-975.

Sallis, J. F., y McKenzie, T. L. (1991). Physical educations' role in public health. Research Quarterly for Exercise and Sport, 62 (2), 124-137.

Sallo, M., y Silla, R. (1997). Physical activity with moderate to vigorous intensity in preschool and first-grade schoolchildren. Pediatric Exercise Science, 9, 44-54.

Sánchez, E. (1990). Hábitos de vida y salud de la población joven de Zaragoza. Tesis Doctoral. Universidad de Zaragoza.

Sánchez, E., García, A. I., y Rubio, E. (1992). Educación en el hábito de la actividad física. Sport \& Medicina, Enero-Febrero, 33-36.

Sánchez-Barrera, M., Pérez, M., y Godoy, J. F. (1995). Patrones de actividad física en una muestra española. Revista de Psicología del Deporte, 7-8, 51-71.

Savage, M.P., Scott, L.B. (1998). Physical activity and rural middle school adolescents. J ournal of Youth and Adolescence, 27 (2), 245-253.

Shannon, B., Peacock, J., y Brown, M. J. (1991). Body fatness, television viewing and calorie-intake of a sample of Pennsylvania sixth grade children. Journal Nutrition Education, 23, 262-268. 
Shepard, J., Jequier, J.C., et al., (1980). Habitual physical activity: effects of sex, milieu, season and required activity. Journal of Sports Medicine, 20, 55-66.

Shepard, R.J., Jéquier, J.C. Lavallée, H., Labarre, R., Rajic, M. (1980). Habitual physical activity: influence of sex, milieu, season and required activity. Journal of Sports Medicine and Physical Fitness, 20, 55-66.

Stephens, T., Jacobs, D.R., White, C.C. (1985). A descriptive epidemiology of leisure time physical activity. Public Health Reports, 100 (2), 147-158.

Taylor, W. C., Blair, S. N., Cummings, S. S., Wun, C. C., y Malina, R, M. (1999). Childhood and adolescent physical activity patterns and adult physical activity. Medicine and Science in Sports and Exercise, 31 (1), 118-123.

Tercedor, P., Delgado, M., Pérez, I., Chillón, P., et al. (2003). Physical activity level in Spanish adolescents. The Avena Study. En II Congreso Mundial de Ciencias de la Actividad Física y el Deporte. Deporte y Calidad de vida. Granada. Noviembre.

Thirlaway, K., Benton, D. (1993). Physical activity in primary and secondary shool children in West Glamorgan. Health Education J ournal, 52 (1), 37-41.

Trost, S., Pate, R. R., Freedson, P. S., Sallis, J. F., y Taylor, W. C. (2000). Using objective physical activity measures with youth: how many days of monitoring are needed? Medicine and Science and in Sports and Exercise, 32 (2), 426-431.

Tucker, L.A. (1990).Television Beijing and physical fitness in adults. Research Quaterly for Exsercise and Sport 61 (4), 315-320.

Verchuur, K.R. Kemper, H.C.G. (1985). The pattern of daily activity. Medicine and Sport Science, 20, 169-186.

Williams, M. (1986). Weight control through exercise and diet for children and young athletes, Effects of physical activity on children. The American Academy of Physical Education Papers, 19, 88-113. Champaign, II: Human Kinetics.

Wold, B. (1989). Lifestyles and Physical Activity. Tesis Doctoral. Norway: University of Bergen. 